\title{
Cuprous Oxide Thin Film Non-Enzymatic Glucose Sensor Using Differential Pulse Voltammetry (DPV) and Other Voltammetry Methods and Comparing to Different Thin Film Electrodes on the Detection of Glucose in an Alkaline Solution
}

\author{
Yifan Dai ${ }^{1}$, Alireza Molazemhosseini ${ }^{2}$, Kevin Abbasi ${ }^{3}$, Chung Chiun Liu ${ }^{1 *}$ \\ ${ }^{1}$ Department of Chemical and Biomolecular Engineering and Electronics Design Center, Case Western \\ Reserve University, 10900 Euclid Ave, Cleveland, OH, 44106, USA; yxd176@case.edu \\ 2Dipartimento di Chimica Materiali ed Ingegneria Chimica "Giulio Natta”, Politecnico di Milano, Via \\ Mancinelli 7, 20131, Italy; axm1058@case.edu \\ ${ }^{3}$ Swagelok Center for Surface Analysis of Materials (SCSAM), Case Western Reserve University, 10900 \\ Euclid Ave, Cleveland, OH, 44106, USA; kxa244@case.edu

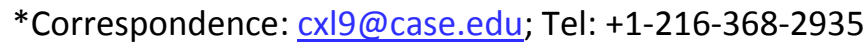

\begin{abstract}
A cuprous oxide $\left(\mathrm{Cu}_{2} \mathrm{O}\right)$ thin layer served as the base for a non-enzymatic glucose sensor in an alkaline medium, $0.1 \mathrm{NaOH}$ solution, with a linear range of $50-200 \mathrm{mg} / \mathrm{dL}$ using differential pulse voltammetry (DPV) measurement. An X-ray photoelectron spectroscopy (XPS) study confirmed the formation of the cuprous oxide layer on the thin gold film sensor prototype. Quantitative detection of glucose in both phosphate-buffered saline (PBS) and undiluted human serum were carried out. Neither ascorbic acid nor uric acid even at a relatively high concentration level of $100 \mathrm{mg} / \mathrm{dL}$ in serum interfered with the glucose detection, demonstrating the excellent selectivity of this non-enzymatic cuprous oxide thin layer based glucose sensor. Chronoamperometry (CA) and single potential amperometric voltammetry were used to verify the measurements obtained by differential pulse voltammetry (DPV), and the positive results validated that the detection of glucose in a $0.1 \mathrm{M} \mathrm{NaOH}$ alkaline medium by DPV measurement was effective. Nickel, platinum and copper are commonly used metals for non-enzymatic glucose detection. The performance of these metal-based sensors for glucose detection using DPV were also evaluated. Cuprous oxide $\left(\mathrm{Cu}_{2} \mathrm{O}\right)$ thin layer based sensor showed the best sensitivity for glucose detection among the sensors evaluated.
\end{abstract}

Key Words: cuprous oxide; non-enzymatic glucose sensor; alkaline solution; differential pulse voltammetry 


\section{Introduction}

An abnormal glucose concentration level is directly related to diabetes, obesity, hyperglycemia and encephalopathy [1]. Therefore, a cost-effective, accurate, consistent glucose sensor is important in medical diagnosis. Measuring glucose concentration is also important for bio-processing and bio-reactor applications, as well as for home care usage. Current glucose sensors are typically based on an enzymatic mechanism with the advantages of low cost and simple operation [2-6]. However, an enzymebased biosensor is limited in accuracy and the reproducibility of measurements are only fair due to the loss of enzyme activity over time. Furthermore, the enzyme also has limited active life time affecting the manufacturing and shelf-life of the glucose sensor. Hence, a cost-effective, highly-accuracy, highlyreproducible, non-enzymatic glucose sensor is desirable for medical applications and bio-processing involving glucose as a reactant or product.

Oxidation of glucose can be detected electrochemically. Metal oxides, polymer films and noble metals have been used as substrate or catalyst for glucose oxidation reaction in non-enzymatic glucose sensor development $[6,7,8]$. Low sensitivity, complex sensor preparation procedures and unpracticality for real usage were the major drawbacks. In this study, copper oxide was used for the detection of glucose in both phosphate-buffered saline (PBS) and human serum. Metal oxides exhibited good electrochemical properties based on their relatively high reactivity and large specific area at nanoscale. However, most of the synthesis and deposition processes of copper oxides were elaborate and tedious, requiring high temperature and relatively long operational time. In this study, electrochemical deposition of cuprous oxide $\left(\mathrm{Cu}_{2} \mathrm{O}\right)$, was accomplished within a relatively short deposition time and with excellent reproducibility. The formation of $\mathrm{Cu}_{2} \mathrm{O}$ was accomplished using $\mathrm{CuSO}_{4} \cdot 5 \mathrm{H}_{2} \mathrm{O}$, lactic acid $\left(\mathrm{C}_{3} \mathrm{H}_{6} \mathrm{O}_{3}\right)$ and $\mathrm{NaOH}$, similar to the preparation approach described by Rahmen [9]. The deposited thin layer $\mathrm{Cu}_{2} \mathrm{O}$ was examined by X-ray photoelectron spectroscopy (XPS) showing high reactivity and reproducibility for both the deposition process and the electrochemical glucose detection.

In the presence of glucose and copper ions from the cuprous or cupric oxide, glucose was oxidized and the copper ions were reduced. The kinetics of the reaction mechanisms were not totally agreed upon, and the assessment of the actual kinetics of copper ions with glucose was beyond the scope of this presentation. Nevertheless, based on reported information of copper (II) oxide for oxidation reaction by hydroxide ions [10,11], copper (I) oxide did demonstrate a high conductivity.

In this study, $\mathrm{Cu}_{2} \mathrm{O}$ was deposited by electrochemical deposition on a thin gold film sensor prototype used in previous studies [12-16]. Glucose concentration ranges of $50-200 \mathrm{mg} / \mathrm{dL}$ in $0.1 \mathrm{M} \mathrm{NaOH}$ solution were detected using differential pulse voltammetry (DPV). Glucose detection was also performed in undiluted human serum using a minute quantity of $0.1 \mathrm{M} \mathrm{NaOH}, 3 \mu \mathrm{L}$ serum containing glucose by DPV. Interference studies by uric acid and ascorbic acid showed that this $\mathrm{Cu}_{2} \mathrm{O}$ based glucose sensor had good selectivity. 


\section{Materials and Methods}

2.1 Apparatus and Reagents

Copper sulfate pentahydrate (\#209198), lactic acid (\#252476), sodium hydroxide (\#306576), D-(+)glucose (\#G-8270), uric acid (\#U-0881), L-ascorbic acid (\#A5960) and human serum (\#H3667) were obtained from Sigma Aldrich (St. Louis, MO). Sodium chloride (\#S-271) was purchased from ThermoFisher (Pittsburgh, PA). Nickel chloride (\#A0282977), and boric acid (\#A0281874) were obtained from Acros Organics, Thermo-Fisher (Pittsburgh, PA). A CHI 660C Electrochemical Workstation (CH Instrument, Inc., Austin, TX,) was used for CV and DPV investigations. Other models of CHI 660 (Models $A-E)$ could also be used. All experiments were conducted at room temperature. $X$-ray photoelectron spectroscopy (XPS) was performed by a PHI (Physical Electronics Inc., Chanhassen, MN.) Versaprobe 5000 Scanning X-Ray Photoelectron Spectrometer using an Aluminum Ka X-ray radiation (50W, 15KV, $1486.6 \mathrm{eV}, 100 \mu \mathrm{m}$ spot size on the sample) served as the excitation source. The analyzer was operated at a constant pass energy of $23.5 \mathrm{eV}$. Under these conditions, the $A u \mathrm{Af}_{7 / 2}$ photoelectron peak was recorded with $0.7 \mathrm{eV}$ at a binding energy of $84.0 \mathrm{eV}$. The calibration of the binding energy of the spectra was performed with the $C 1 \mathrm{~s}$ peak of the adventitious carbons, which was at $284.8 \mathrm{eV}$. The spectra of each sample was obtained with a short acquisition time of 10 minutes to examine $\mathrm{C} 1 \mathrm{~s}, \mathrm{Cu} 2 \mathrm{p}$ and $\mathrm{Cu}$ LMM XPS regions in order to avoid, as much as possible, the photo-reduction of species. The information from the outermost $\sim 10 \mathrm{~nm}$ of the surface was converted to a depth profile using data acquired in an angular dependent XPS experiment. When the angle between sample normal and the analyzer entrance was increased, with the X-ray source and analyzer kept in fixed positions, the photoelectrons originated from an increasingly surface localized zone. Spectrums were acquired at takeoff angles of $0^{\circ}, 45^{\circ}$ and $80^{\circ}$ in order to obtain information about the composition as a function of the depth.

The electrochemical methods used in this study were described separately in Section 3 below.

\subsection{Fabrication of Non-enzymatic $\mathrm{Cu}_{2} \mathrm{O}$ Glucose Sensor}

Sputtered thin gold film sensor prototype used in this study had been described elsewhere [12-16]. Electrochemical deposition of the cuprous oxide film on the thin gold film working sensor element was carried out. A mixture of $0.2 \mathrm{M}$ cupric sulfate, $3 \mathrm{M}$ of lactic acid and approximately $3 \mathrm{M}$ of sodium hydroxide for adjusting $\mathrm{pH}$ value to 12 was used as electrolyte in this $\mathrm{Cu}_{2} \mathrm{O}$ deposition $[9,17]$. A water bath was used for maintaining the electrolyte solution at $40^{\circ} \mathrm{C} .20 \mu \mathrm{L}$ of prepared electrolyte solution was casted on the sensor and linear sweep voltammetry was applied for deposition of cuprous oxide. Linear sweep voltammetry of potential from $-0.8 \mathrm{~V}$ to $-0.1 \mathrm{~V}$ was applied for deposition. Electrochemical deposition potential was at $-0.36 \mathrm{~V}$ versus the thick-film printed $\mathrm{Ag} / \mathrm{AgCl}$ reference electrode. The darker color of the working electrode (in the center of the sensor prototype) shown in Figure 1 shows the 
deposition of the cuprous oxide layer on the thin gold film working electrode. After deposition, the cuprous oxide thin film sensor was washed with DI water, dried by nitrogen and ready for use.

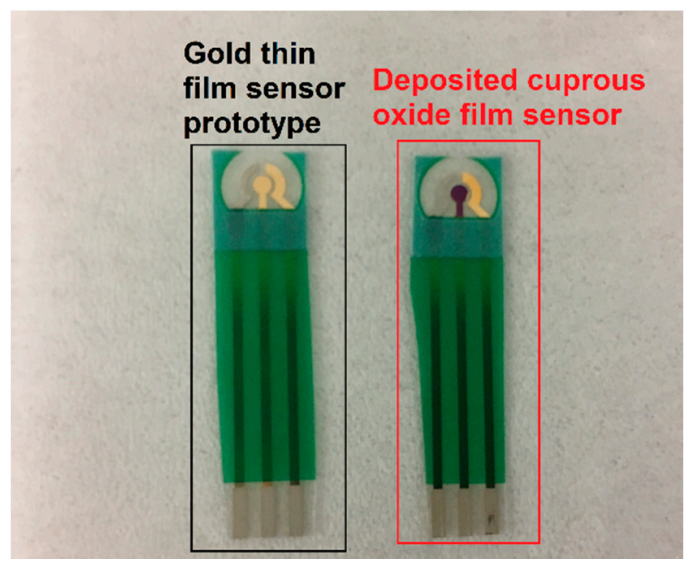

Figure1. Comparison of gold sensor prototype and prepared cuprous oxide film sensor.

\section{Results and Discussion}

\subsection{Surface Characterization of Cuprous Oxide Layer with XPS}

X-ray photoelectron spectroscopy (XPS) was used for examining the formation of cuprous oxide film on the thin gold film working electrode. The chemical shift of the $\mathrm{Cu} 2 \mathrm{p} 3 / 2$ photoelectron peak was not detectable with the energy resolution of the XPS. The presence and the intensity of the satellite peaks for the kinetic energy was at 940-945 eV region, indicating a binding energy of $570 \mathrm{eV}$. The peak shape and position of $\mathrm{Cu}$ LMM were experimentally verified and matched with the reference data [18]. The experimental data from 2 different samples along with the reference data are shown in Figure 2 . The decreasing of the take-off angle increased the height of the Cu2p due to the smaller of the take-off angle resulting in the sample became closer to the detector and with more photoelectrons reaching the detector. Consequently, the results also confirmed the presence of $\mathrm{Cu}_{2} \mathrm{O}$ by forming weak satellites in between $\mathrm{Cu} 2 \mathrm{p} 3 / 2$ and $\mathrm{Cu} 2 \mathrm{p} 1 / 2$ peaks. This conclusion was supported from the spectra acquired at various take-off angles proving that a uniform $\mathrm{Cu}_{2} \mathrm{O}$ layer was formed.
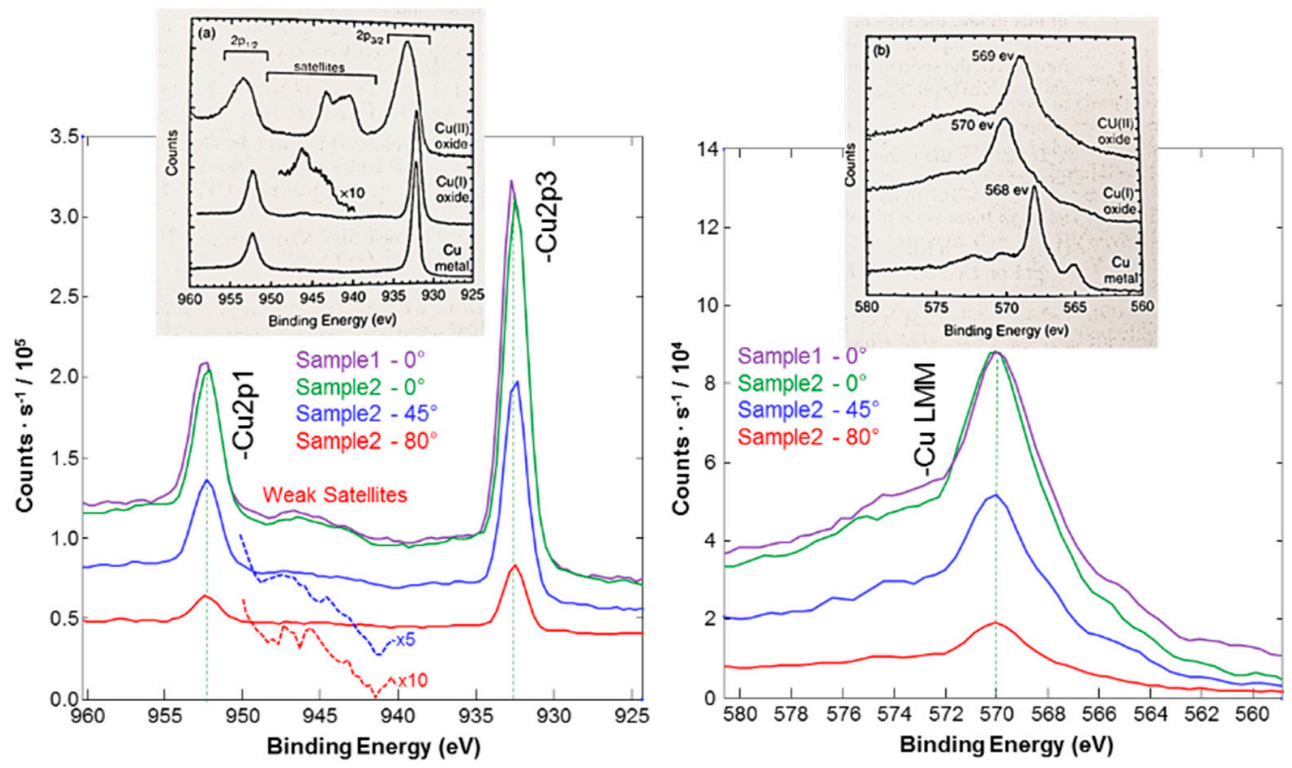
Figure 2. High resolution XPS spectrums of Copper photoelectron peaks compared on two different samples. The take-off angle on one of the samples verified the consistency of the measurement at different thicknesses.

\subsection{Electrochemical Measurement of Glucose by Differential Pulse Voltammetry (DPV)}

Glucose was prepared in a $0.1 \mathrm{M} \mathrm{NaOH}$ solution with the concentration ranging from $50 \mathrm{mg} / \mathrm{dL}$ to 200 $\mathrm{mg} / \mathrm{dL}$. Based on the mechanism of reaction of glucose with cuprous oxide in alkaline solution, an increase of the anodic peak current at a potential of approximately $+0.4 \mathrm{~V}$ versus a thick-film printed $\mathrm{Ag} / \mathrm{AgCl}$ reference electrode was observed with increasing concentration of glucose. In a typical experimental run, $20 \mu \mathrm{L}$ of $0.1 \mathrm{M} \mathrm{NaOH}$ with known glucose solution was drop-casted on the cuprous oxide based sensor. A rest time was set for $10 \mathrm{~s}$ allowing the hydroxide ion to first oxidize the cuprous oxide. DPV was then conducted in the range of 0 to $+0.75 \mathrm{~V}$ versus the thick-film printed $\mathrm{Ag} / \mathrm{AgCl}$ reference electrode. DPV measurements of different glucose concentrations in $0.1 \mathrm{M} \mathrm{NaOH}$ solution are shown in Figure 3a. The calibration curve based on the DPV current output and concentration of glucose is shown in Figure $3 b$. A linear relationship $Y=0.024 X+1.46$ with adjusted $R$ square value of $0.978(n>5)$ is established demonstrating excellent sensitivity and reproducibility of cuprous oxide film based glucose sensor.

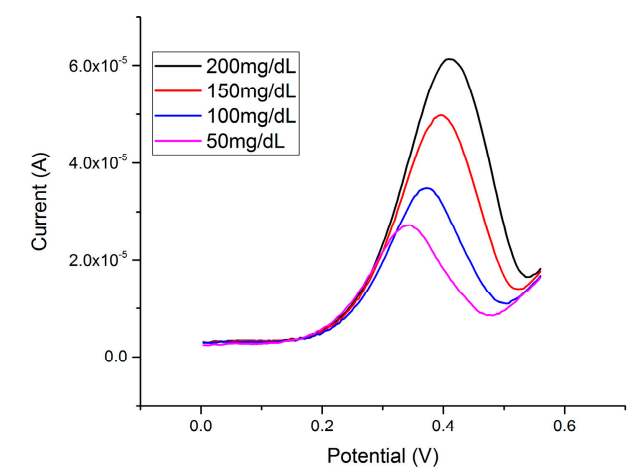

Figure 3a. DPV measurements of glucose concentrations ranging from $200 \mathrm{mg} / \mathrm{dL}$ to $50 \mathrm{mg} / \mathrm{dL}$.

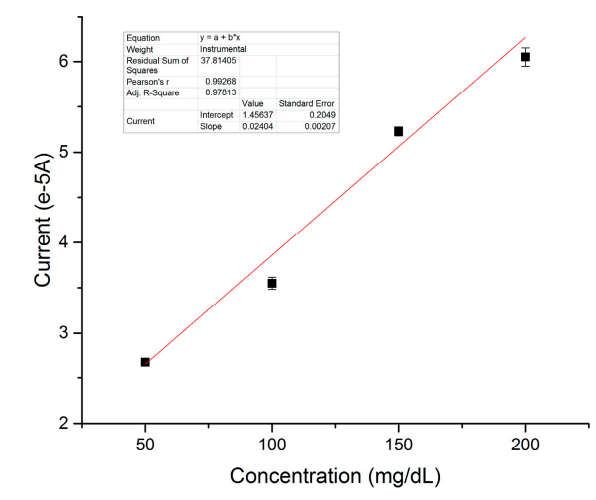

Figure 3b. Calibration linear relationship of DPV current outputs and concentrations of glucose. 


\subsection{Glucose detection by Chronoamperometry (CA) and Single-potential Amperometric Voltammetry}

Determination of glucose using a $\mathrm{Cu}_{2} \mathrm{O}$ thin layer based sensor and DPV measurement demonstrated that the detection technique was very effective. However considering the electrochemical complexity of DPV compared to commonly used CA and single-potential amperometric voltammetry, glucose detection by $\mathrm{CA}$ and single-potential amperometric voltammetry were also carried out. The results validated that $\mathrm{Cu}_{2} \mathrm{O}$ thin layer based sensor for glucose detection with different electrochemical detection techniques in addition to DPV measurement was successful. Furthermore, the results of this study verified the effectiveness of $\mathrm{a} \mathrm{Cu}_{2} \mathrm{O}$ thin layer based sensor for glucose detection in an alkaline medium. Figure 4 shows the chronoamperometry (CA) response of a $\mathrm{Cu}_{2} \mathrm{O}$ thin layer based sensor to different glucose concentration in $0.1 \mathrm{M} \mathrm{NaOH}$ test medium. The testing procedure was the same as mentioned in section 3.2. In this CA measurement, a voltage of $+0.35 \mathrm{~V}$ versus $\mathrm{Ag} / \mathrm{AgCl}$ reference was applied and then with a step change of potential to $+0.4 \mathrm{~V}$ in voltage.

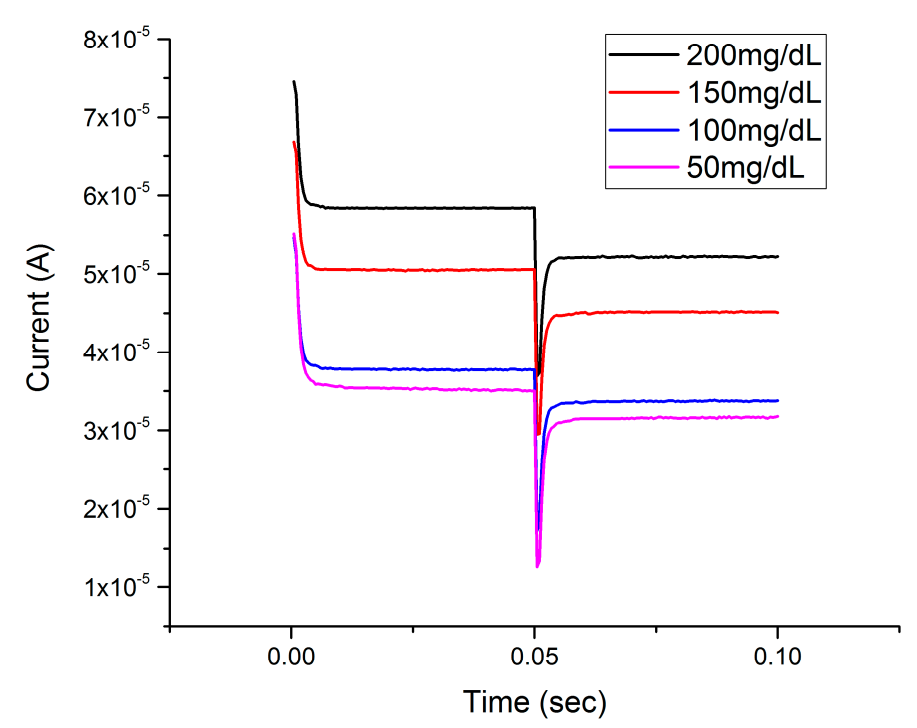

Figure 4. Chronoamperometry measurement of glucose concentrations ranging from $200 \mathrm{mg} / \mathrm{dL}$ to $50 \mathrm{mg} / \mathrm{dL}$.

Figure 5 shows the single-potential amperometric voltammetry responses on glucose concentrations ranging from $50 \mathrm{mg} / \mathrm{dL}$ to $200 \mathrm{mg} / \mathrm{dL}$ in $0.1 \mathrm{M} \mathrm{NaOH}$ solution. There was no potential step change in single-potential amperometric voltammetry, compared to CA measurement. Thus, this cuprous oxide film based sensor also showed an excellent response in the single-potential amperometric voltammetry at a single potential of $+0.5 \mathrm{~V}$ versus $\mathrm{Ag} / \mathrm{AgCl}$ reference electrode. A rest time of $10 \mathrm{~s}$ was used allowing 
the reaction between glucose and $\mathrm{Cu}_{2} \mathrm{O}$ to reach a steady state. The single-potential amperometric voltammetry took $0.3 \mathrm{~s}$ to complete.

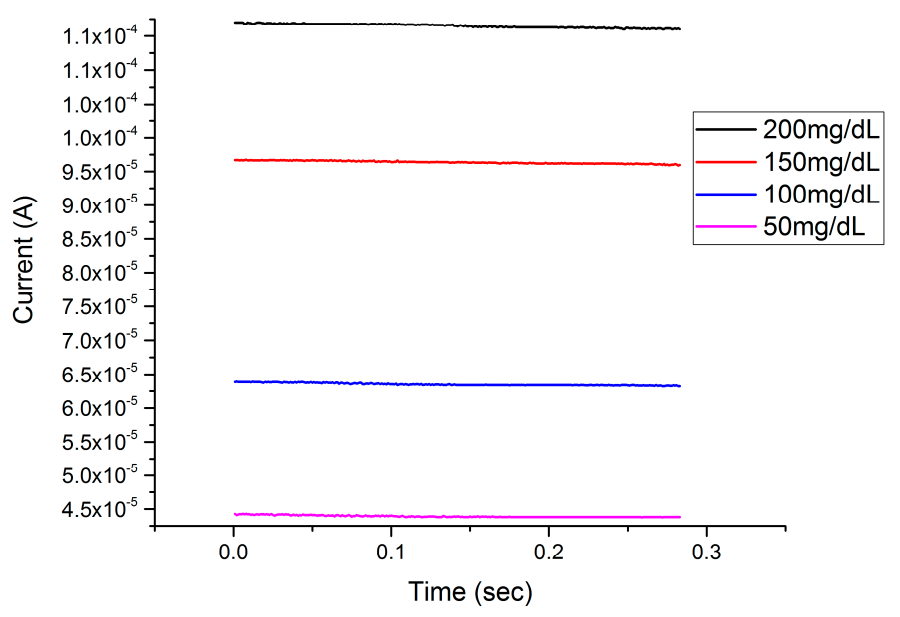

Figure 5. Single-potential amperometric voltammetry measurement of glucose concentrations ranging between $50 \mathrm{mg} / \mathrm{dL}$ and $200 \mathrm{mg} / \mathrm{dL}$

The detection responses of both chronoamperometry and single-potential amperometric voltammetry as demonstrated in Figures 4 and 5 provided verification of the detection of glucose of a $\mathrm{Cu}_{2} \mathrm{O}$ thin layer based sensor in an alkaline medium by DPV technique.

\subsection{Detection of Glucose in Undiluted Human Serum by DPV}

In a typical run, $3 \mu \mathrm{L}$ of glucose in serum solution was mixed with $3 \mu \mathrm{L}$ of $0.1 \mathrm{M} \mathrm{NaOH}$ solution. Then, this $6 \mu \mathrm{L}$ of the mixed solution was placed on the sensor and DPV was applied as described in section 3.2. Figure 6a shows the DPV detection responses of the sensor for glucose solutions in human serum ranging from $50 \mathrm{mg} / \mathrm{dL}$ to $200 \mathrm{mg} / \mathrm{dL}$. Figure $6 \mathrm{~b}$ shows the calibrated linear fit for the DPV results with an equation of $Y=0.016 X+0.847$ and adjusted $R$ square value of $0.929(n>5)$.

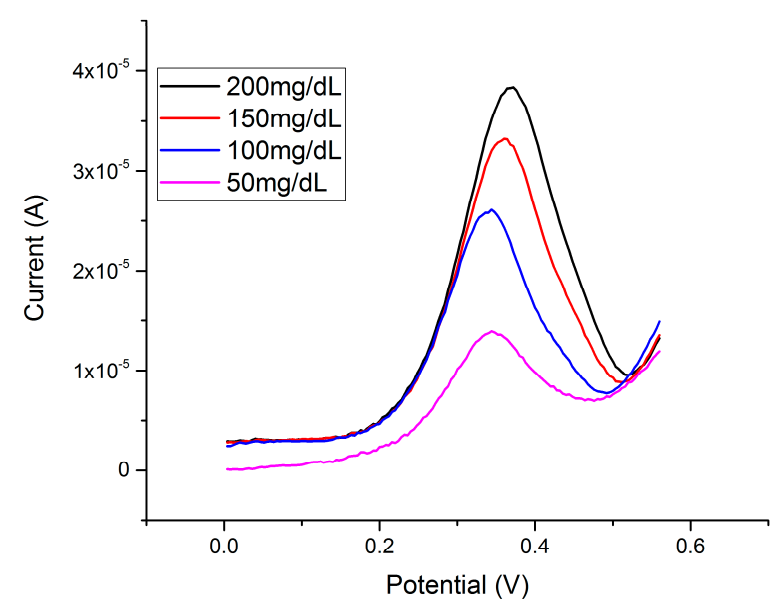


Figure 6a. Differential pulse voltammetry measurement of glucose concentrations ranging from 50 $\mathrm{mg} / \mathrm{dL}$ to $200 \mathrm{mg} / \mathrm{dL}$ in undulated human serum.

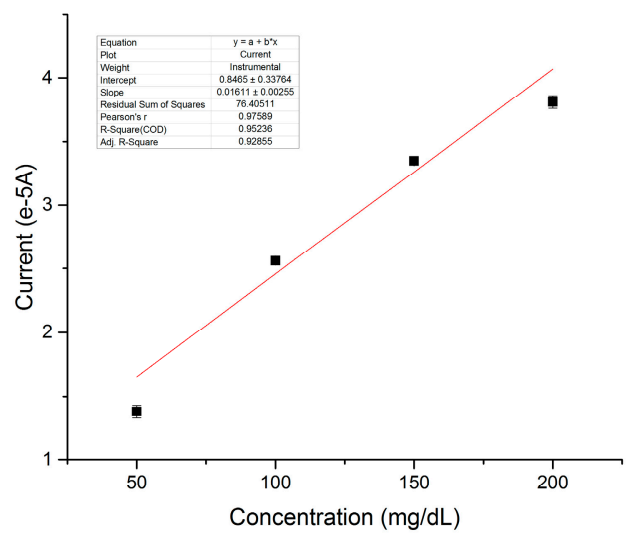

Figure 6b. Calibration linear relationship of DPV current outputs and concentrations of glucose.

DPV measurements demonstrated that this $\mathrm{Cu}_{2} \mathrm{O}$ thin layer based glucose sensor could be used effectively in blood serum by adding a minor volume of $3 \mu \mathrm{L}$ of $0.1 \mathrm{M} \mathrm{NaOH}$ to the glucose test sample. This $3 \mu \mathrm{L}$ of $0.1 \mathrm{M} \mathrm{NaOH}$ or similar hydroxide ion containing solutions could be applied to the glucose test sample in serum with minimum inconvenience.

\subsection{Interference Study of $\mathrm{Cu}_{2} \mathrm{O}$ Thin Layer Based Sensor for Glucose Detection}

Interference testing was important to ensure the selectivity of the $\mathrm{Cu}_{2} \mathrm{O}$ thin layer based sensor for glucose detection. Two common inference chemicals of glucose sensing, ascorbic acid and uric acid, were used in this study. The actual quantities of these interfering species are relatively minute compared with the quantity of glucose in human blood [19]. However, a relatively large quantity of ascorbic acid or uric acid was used in this interference study demonstrating that the selectivity of this $\mathrm{Cu}_{2} \mathrm{O}$ thin layer based glucose sensor was excellent. $100 \mathrm{mg} / \mathrm{dL}$ of ascorbic acid and $100 \mathrm{mg} / \mathrm{dL}$ of uric acid were prepared individually in undiluted human serum. The same testing protocol described in section 3.4 was conducted. Both ascorbic acid and uric acid at this high concentration level did not contribute any current in the DPV measurement of glucose detection as shown Figure 7. The results demonstrated that the selectivity of this $\mathrm{Cu}_{2} \mathrm{O}$ thin layer based sensor for glucose sensing in an alkaline medium was excellent. 


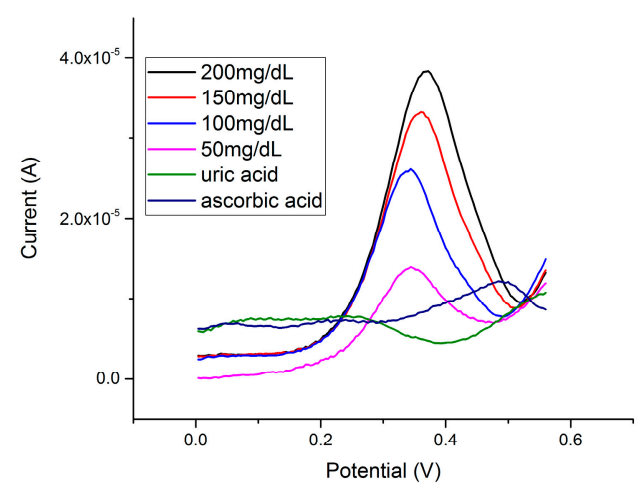

Figure 7. Interference tests from uric acid and ascorbic acid performed by DPV.

3.6 Non-enzymatic Metallic Catalyst Based Glucose Sensors in Alkaline Solution

In additional to cuprous oxide thin film, metallic catalysts, such as nickel, platinum, and copper, also showed promising ability in reaction with glucose in an alkaline condition [4, 5, 19-21]. The reaction mechanism demonstrated that the alkaline solution oxidized metal catalysts, then glucose reduced the oxidized metal producing gluconic acid [4, 5, 14, 19, 21]. Thus, the performance of metallic catalysts, including nickel, platinum and copper for detection of glucose in alkaline solution, were examined and compared with the cuprous oxide thin film sensor.

\subsubsection{Electrochemical Deposited Copper Film for the Detection of Glucose in Alkaline Solution}

Copper was used for the detection of organic compounds based on its oxidation activity in alkaline solution [21], including using cuprous and cupric oxides as discussed above. In order to assess the effect and the role of copper serving as a metal based sensor for glucose detection, copper was electrochemically deposited onto the thin gold film sensor prototype and evaluated. Cathodic reduction of $\mathrm{Cu}^{+2}$ ions from an electrolyte was employed for the electrochemical deposition of copper onto the thin gold film sensor prototype. Typically, an electrolyte of $0.05 \mathrm{M}$ of $\mathrm{CuSO}_{4}$ and $0.1 \mathrm{M}$ of $\mathrm{H}_{2} \mathrm{SO}_{4}$ in aqueous solution was used for the copper deposition. $20 \mu \mathrm{L}$ of the electrolyte was placed on the gold thin film sensor and linear sweep voltammetry of $-0.9 \mathrm{~V}$ to $-0.3 \mathrm{~V}$ was applied for deposition of copper at room temperature. Cathodic peak current at $-0.86 \mathrm{~V}$ versus $\mathrm{Ag} / \mathrm{AgCl}$ reference electrode was observed for this reduction reaction. The protocol of detecting of glucose using this copper based sensor was identical to the evaluation of other metal or metal catalyst based glucose sensors as described in section 3.2. DPV was applied, and the oxidation reaction between glucose and copper took place at around $+0.40 \mathrm{~V}$ vs. $\mathrm{Ag} / \mathrm{AgCl}$ as reference electrode. Figure $8 \mathrm{a}$ shows the anodic currents of the DPV measurements of glucose concentrations of $50-200 \mathrm{mg} / \mathrm{dL}$ in a $0 . \mathrm{i} \mathrm{M} \mathrm{NaOH}$ solution. The anodic peak currents appeared at approximately $+0.40 \mathrm{~V}$ versus the thick-film printed $\mathrm{Ag} / \mathrm{AgCl}$ reference electrode.

\subsubsection{Electrochemical deposited nickel film for detection of glucose in basic solution}

Nickel was a good biological reaction catalyst with a significant chemical activity [20]. Nickel was an active material for glucose detection in the presence of $\mathrm{OH}^{-}[21,22]$. The performance of nickel on the detection of glucose was evaluated and compared to the cuprous oxide thin film layer based sensor for glucose detection. Nickel was deposited electrochemically onto the thin gold film sensor prototype. An electrolyte containing $0.14 \mathrm{M} \mathrm{NiCl}_{2}, 1 \mathrm{M} \mathrm{NaCl}, 0.5 \mathrm{M} \mathrm{H}_{3} \mathrm{BO}_{3}$ and a copious amount of $\mathrm{HCl}$ for adjusting the 
solution $\mathrm{pH}$ value to around 1.5 was prepared for the deposition of nickel [23]. $20 \mu \mathrm{L}$ of an electrolyte was placed on the gold thin film sensor and linear sweep voltammetry of $-1.2 \mathrm{~V}$ to $-0.7 \mathrm{~V}$ was applied for deposition of nickel at room temperature. An increasing reduction cathodic deposition peak was observed at $-1 \mathrm{~V}$ vs. $\mathrm{Ag} / \mathrm{AgCl}$ as reference electrode. The procedure of detection of glucose in an alkaline solution using this nickel based sensor was identical to the process described in section 3.2. Differential pulse voltammetry was applied and an anodic peak was obtained at $+0.38 \mathrm{~V}$ versus the thick film printed $\mathrm{Ag} / \mathrm{AgCl}$ reference electrode [13,14,16], which was also confirmed by Luo's study [21]. Figure 8b shows the differential pulse voltammetry graph for the detection of glucose in $0.1 \mathrm{M} \mathrm{NaOH}$ solution using the electrochemically deposited nickel thin film sensor covering the glucose concentration range of $50 \mathrm{mg} / \mathrm{dL}$ to $200 \mathrm{mg} / \mathrm{dL}$.

\subsubsection{Sputtered Thin Platinum Film Sensor for the Detection of Glucose in Alkaline Solution}

Platinum is well-accepted as a bioactive metal or catalyst for the detection of organic compounds, including carbohydrates, amino acids and glucose [21]. Therefore, the detection of glucose using a platinum based sensor was also studied in this research endeavor. The fabrication of the thin platinum film sensor prototype was identical to the process of the thin gold film sensor prototype [12-16] with the only difference that platinum (50nm thickness) was used for the working and the counter electrodes stead of gold. Sputtering physical vapor deposition, laser ablation and thick-film printing technologies were used, and the platinum thin film sensor could also be fabricated by roll-to-roll cost-effective manufacturing process. The testing protocol of glucose using this platinum thin film based sensor was identical to the testing procedure described in section 3.2. DPV was applied and anodic peak currents for different glucose concentrations were observed at approximately $+0.43 \mathrm{~V}$ versus the thick-film printed $\mathrm{Ag} / \mathrm{AgCl}$ reference electrode. Figure $8 \mathrm{c}$ shows the DPV measurements of various glucose concentrations of glucose in $0.1 \mathrm{M} \mathrm{NaOH}$ solution ranging from $50 \mathrm{mg} / \mathrm{dL}$ to $200 \mathrm{mg} / \mathrm{dL}$.

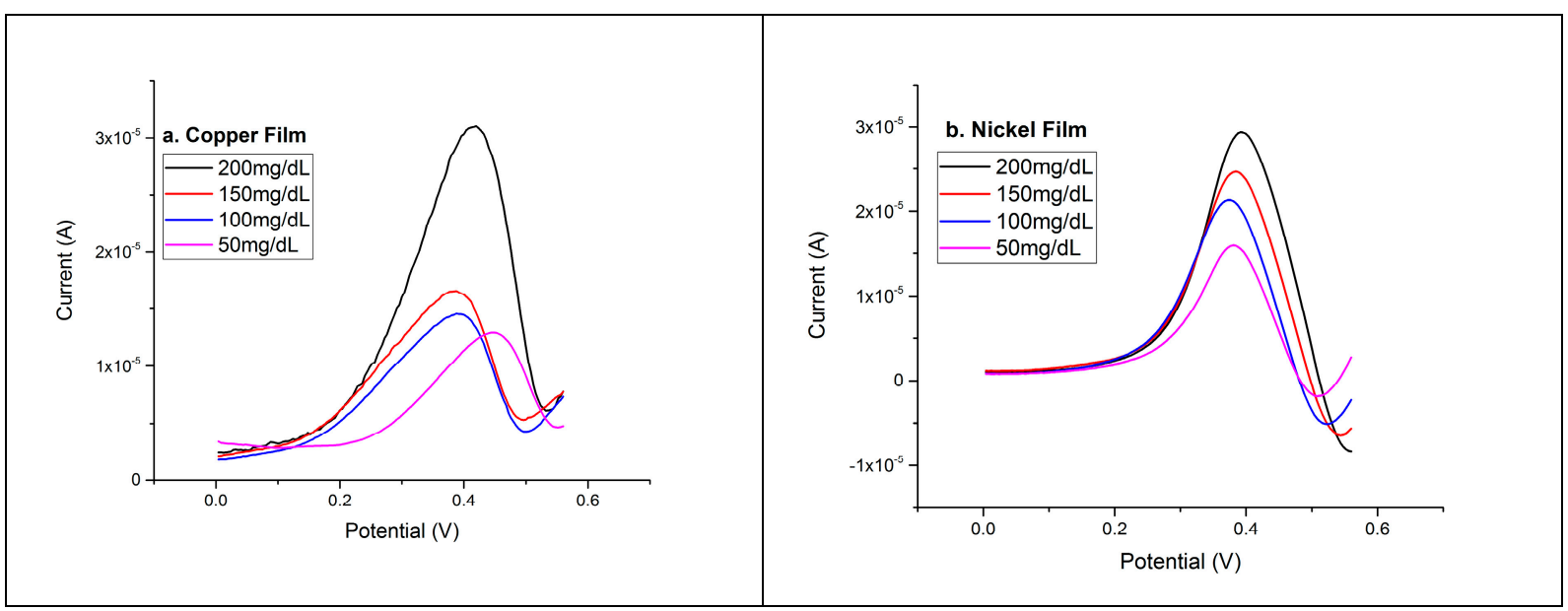




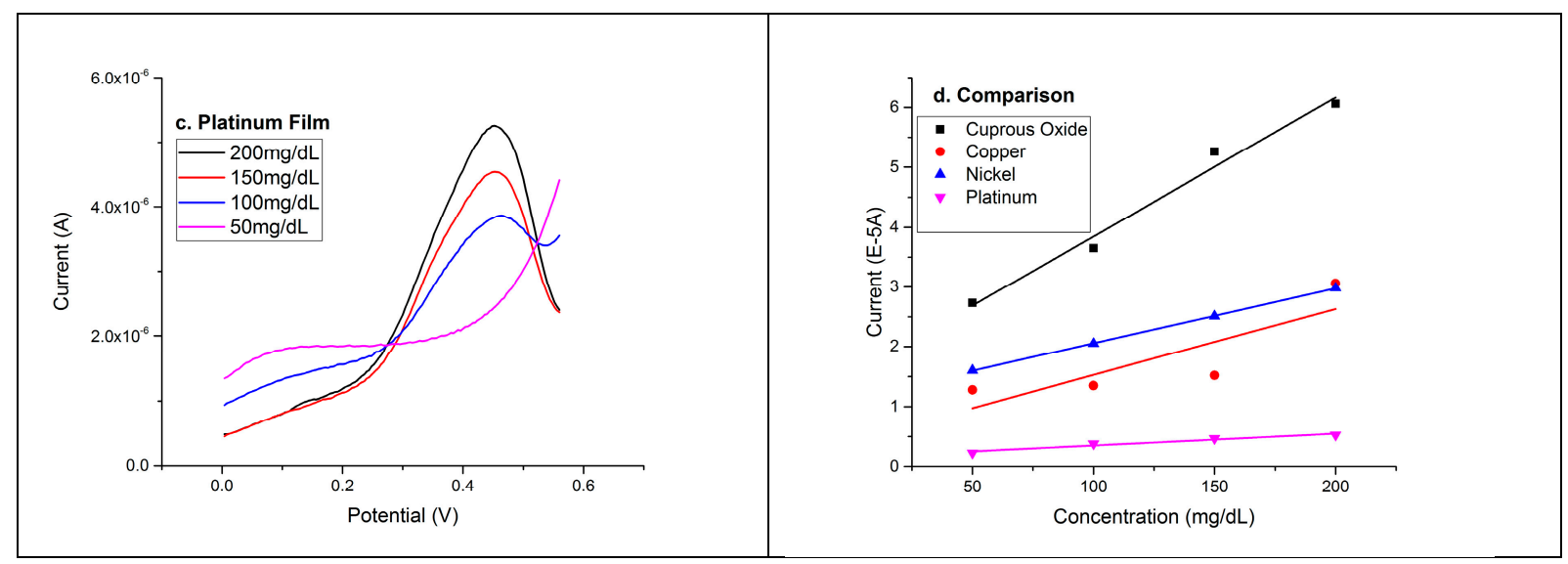

Figure 8. Detection response based on different metal films (a. copper, b. nickel, c. platinum, d. comparison).

This section 3.6 discussed the experimental performance of various metals as catalysts for glucose detection using DPV measurements. Figure $8 \mathrm{~d}$ summarized the performance of these non-enzymatic glucose sensors by displaying their calibration curves based on the data acquired. Compared with metal films sensor for detection of glucose in alkaline solution, cuprous oxide demonstrated the highest current outputs, showing a promising application as a non-enzymatic glucose sensor.

\section{Conclusions}

A non-enzymatic cuprous oxide $\left(\mathrm{Cu}_{2} \mathrm{O}\right)$ thin layer based sensor for the detection of glucose in an alkaline medium, $0.1 \mathrm{NaOH}$ solution over the glucose concentration range of 50-200 mg/dL was successfully developed using differential pulse voltammetry (DPV) measurement. X-ray photoelectron spectroscopy (XPS) confirmed the formation of cuprous oxide, $\mathrm{Cu}_{2} \mathrm{O}$ layer on the thin gold film sensor prototype. The evaluation of glucose in both phosphate-buffered saline (PBS) and undiluted human serum were carried out. The $0.1 \mathrm{M} \mathrm{NaOH}$ alkaline solution used was minute, $3 \mu \mathrm{L}$ in a total of $6 \mu \mathrm{L}$ test medium. Neither ascorbic acid nor uric acid, even at a high concentration level of $100 \mathrm{mg} / \mathrm{dL}$ in serum, interfered with the cuprous oxide ( $\mathrm{Cu} 2 \mathrm{O}$ ) thin layer based sensor in glucose measurement., demonstrating the selectivity of this non-enzymatic cuprous oxide $\left(\mathrm{Cu}_{2} \mathrm{O}\right)$ thin layer based sensor was excellent. Chronoamperometry (CA) and single-potential amperometric voltammetry were also used in the glucose detection experimentally using this cuprous oxide $\left(\mathrm{Cu}_{2} \mathrm{O}\right)$ thin layer based sensor. The positive results verified the validity of detecting glucose in a $0.1 \mathrm{M} \mathrm{NaOH}$ alkaline medium by DPV measurement. Nickel, platinum and copper were commonly used metals for non-enzymatic glucose detection. The performance of these metal based sensors for glucose detection using DPV technique were experimentally evaluated. Cuprous oxide $\left(\mathrm{Cu}_{2} \mathrm{O}\right)$ thin layer based sensor showed the best sensitivity for glucose detection among the sensors evaluated.

Acknowledgments: The authors would like to acknowledge Swagelok Center for Surface Analysis of Materials (SCSAM) of Case Western Reserve University (CWRU) providing facility and technical assistance in the XPS characterization in this study. Alireza Molazemhosseini, gratefully acknowledges Italian Ministry of Education, Universities and Research (MIUR) for his PhD scholarship and Electronics Design Center of CWRU for their financial support. Financial support by Wallace R. Persons research fund from Case Alumni Association (CAA) for this research is also acknowledged. 
Author Contributions: C.C.L., A.M. and Y.D. conceived and designed the experiments; Y.D. A.M. K.A. performed the experiments; C.C.L., A.M. Y.D. K.A. analyzed the data; Y.D., A.M. K.A. and C.C.L. all contributed to prepare this manuscript.

\section{References}

[1] http://www.medicinenet.com/encephalopathy/article.htm

[2] Zhang M.; et al. Highly sensitive glucose sensors based on enzyme-modified whole-graphene solution-gated transistors. Scientific reports (2015) 5, 8311.

[3] Tian K.; Alex S.; Siegal G.; Tiwari A. Enzymatic glucose sensor based on Au nanoparticle and plant-like ZnO film modified electrode. Materials Science and Engineering: C (2015), 46, 548-552.

[4] Jiang LC.; Zhang WD. A highly sensitive nonenzymatic glucose sensor based on CuO nanoparticlesmodified carbon nanotube electrode. Biosens. Bioelectron. (2010) 25, 1402-1407.

[5] Reitz E.; et al. CuO nanospheres based nonenzymatic glucose sensor, Electroanalysis (2008),20, 2482-2486.

[6] Li LH.; Zhang WD. Preparation of carbon nanotubes supported platinum nanoparticles by an organic colloidal process for nonenzymatic glucose sensing. Microchimica Acta (2008), 163, 305- 311.

[7] Sun YP.; Buck H.; Mallouk TE. Combinatorial discovery of alloy electrocatalysts for amperometric glucose sensors. Analytical Chemistry (2001), 73, 1599-1604.

[8] Espro C.; et al. CuO nanowires-based electrodes for glucose sensors. Chemical Engineering Transactions (2014), 41, 415-420.

[9] Rahman A S.; Shorowordi K M. Electrodeposition and characterization of copper oxide thin films for solar cell applications. Procedia Engineering (2015), 105, 679-685.

[10] Torto N.; Ruzgas T.; Gorton L. Electrochemical oxidation of mono- and disaccharides at fresh as well as oxidized copper electrodes in alkaline media, J. Electroanal. Chem.(1999) 464, 252-258,

[11] Corbo D.; Bertotti M. Amperometric determination of ethanol in beverages at copper electrodes in alkaline medium, Anal. Chim. Acta (2002), 472,123-131,

[12] Dai, Y.; Liu, C.C. A simple, cost-effective sensor for detecting lead ions in water using underpotential deposited bismuth sub-layer with differential pulse voltammetry (DPV). Sensors (2017), 17, 950.

[13] Dai, Y.; Molazemhosseini, A.; Liu, C.C. In vitro quantified determination of $\beta$-amyloid 42 peptides, a biomarker of neuro-degenerative disorders, in PBS and human serum using a simple, cost-effective thin gold film biosensor. Biosensors (2017), 7, 29.

[14] Molazemhosseni, A.; Magagnin, L.; Vena, P.; Liu, C.C. Single-use nonenzymatic glucose biosensor based on $\mathrm{CuO}$ nanoparticles ink printed on thin film gold electrode by micro-plotter technology. Journal of Electroanalytical Chemistry (2017),789, 50-57. 
[15] Dai, Y.; Liu, C.C. Detection of $17 \beta$-Estradiol in environmental samples and for health care using a single-use, cost-effective biosensor based on differential pulse voltammetry (DPV). Biosensors (2017), 7, 15.

[16] Dai, Y.; Molazemhosseini, A.; Liu, C.C. A single-use, in vitro biosensor for the detection of T-tau protein, a biomarker of neuro-degenerative disorders, in PBS and human serum using differential pulse voltammetry (DPV). Biosensors (2017), 7, 10.

[17] Du, QT.; et al. Electrochemical deposition and formation mechanism of single-crystalline $\mathrm{Cu}_{2} \mathrm{O}$ octahedral on aluminum. Journal of Analytical Methods in Chemistry (2012), 406162.

[18] Surface Analysis: The principal techniques $2^{\text {nd }}$ edition by John Vickerman and lan Gilmore Wiley Publications, ISBN 978-0-470-01763-0 (2009)

[19] Zhou C,; Xu L,; Song J,; Xing R,; Xu S,; Liu D,; Song H. Ultrasensitive non-enzymatic glucose sensor based on three-dimensional network of $\mathrm{ZnO}-\mathrm{CuO}$ hierarchical nanocomposites. Scientific Reports(2014), 4:7382.

[20] Zamble D. Nickel in biology. Royal society of chemistry (2015) 7, 588.

[21] Luo P.; Zhang F.; Baldwin R. Comparison of metallic electrodes for constant-potential amperometric detection of carbohydrates, amino acids and related compounds in flow systems. Analytica Chimica Acta (1991), 244, 169-178.

[22] Fleischmann M.; Korinek K.; PletcherD. The oxidation of organic compounds at a nickel anode in alkaline solution. J. Electroanal. Chem (1971), 31, 39.

[23] Boubatra M.; Azizi A.; Schmerber G.; Dinia A. The influence of pH electrolyte on the electrochemical deposition and properties of nickel thin films. Ionics (2012), 18, 4, 425-432. 